
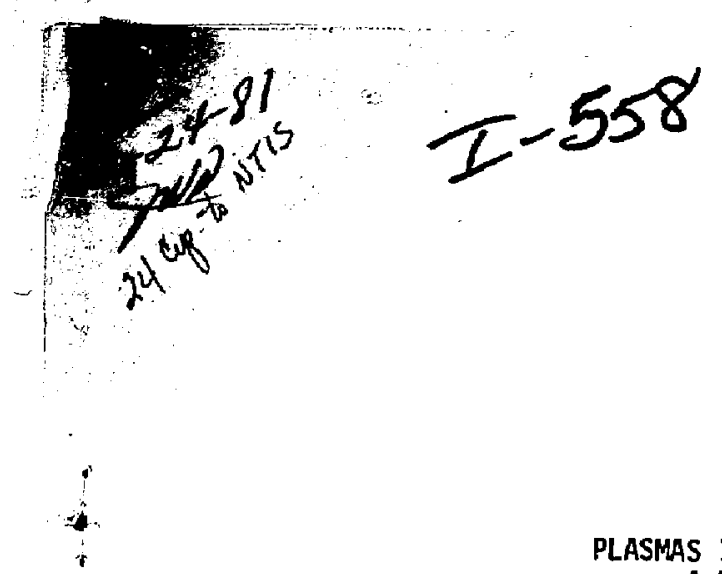

(1)

UCID--18947 Pt. 3 Rev. 1

DE82 006182

\title{
PLASMAS IN PARTICLE ACCELERATORS: \\ A HYOROQYMAMIC MODEL OF THREE-DIMENSIONAL ELECTROSTATIC INSTABILITIES
}

James W-K. Mark, Geoffrey A. Krafft, and Taj-Sen F. Wang

December 1, 1981

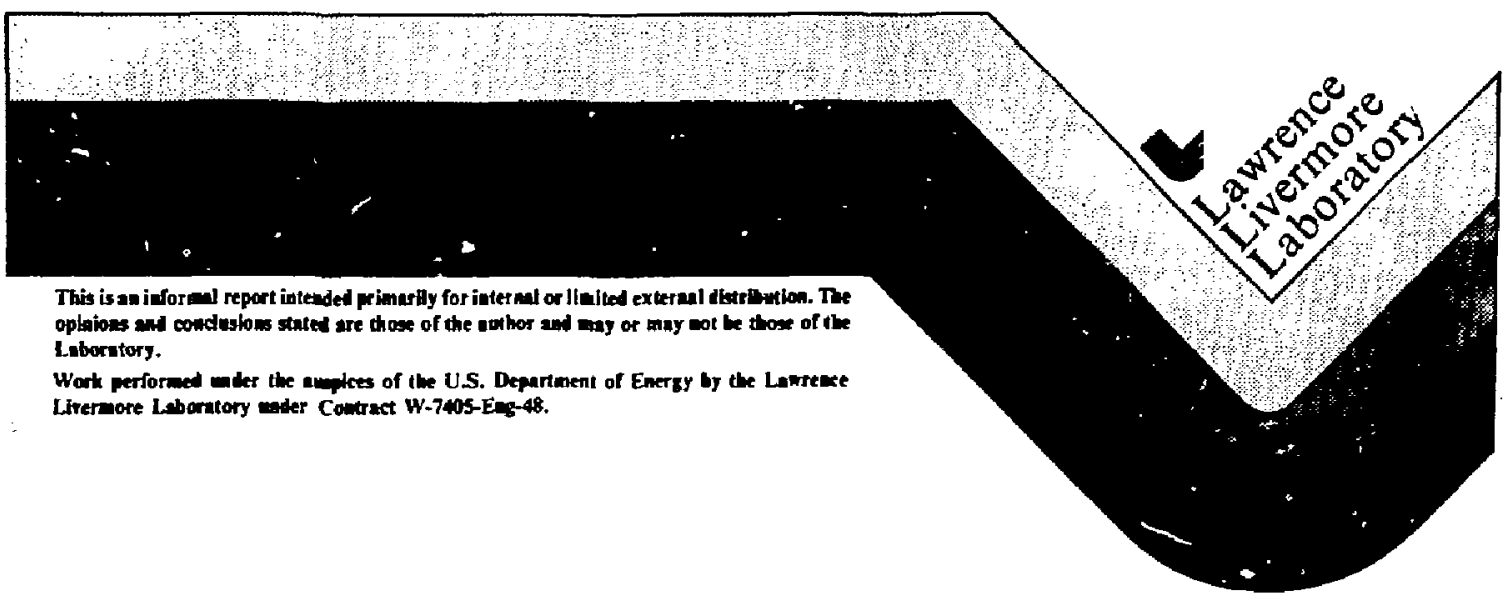

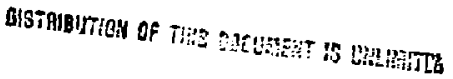




\title{
ABSTRACT
}

\begin{abstract}
A hydrodynamic model is used to help isolate possible three dimensional space charge instabilities in beam plasmas of concern in aesigning heavy ion accelerators for inertial confinement fusion energy apilications. The model provides an economic means for searching the large para eter space relevant to problenis in which coupling of longituainal and transver $\$$ - motions is allowed. It is shown that the equilibrium axial hydrodynamic pressure of the beam plasma has a significant effect on the stability boundaries of a two-rotating-stream instability. When considering the resistive wall effect, this model shows a kink instability. The growth rate of some modes could be enhanced by increasing the equilibrium axial pressure.
\end{abstract}


I. INTRODUCTION

Induction linear particle accelerators are being designed for application as drivers for heavy ion inertial fusion energy power plants [ $1-4]$. As part of the process, it is necessary to identify and avoid regimes of possible ihree-dimensional instabilities which involve coupling of longitudinal and transverse motions under anharmonic space charge forces which are now no longer negligible. This task has been considered prohibitive in the past partly because of the three spatial dimensions and partly because of the number uf potential parameters involved in its solution. (E.g. even in the simplified discussion of Section III, there are nine parameters). A further hindrance is the fact that the realistic final discussion must involve the kinetic Vlasov description (cf. Ref. 5) in six-dimensional phase space and time with non-axisymmetric forces. Three dimensional particle codes with self-consistent space charge forces could in principle be used out are not realistic as a first attempt because of the computational expense needed to survey the extensive parameter space.

The above three aimensional problen is made more tractable to analytical efforts through approximations introduced by Mark and Smith [6; hereinafter referred to as Paper []. Their scheme involved an ion beam subject to focusing forces which to the first approximation are axisymmetric. Thus we assume that the forces on an initially axisymetric beam (Fig. 1) can be separated into a dominant averaged part $F_{a v}(r, \varepsilon z, \epsilon t)$ and a fluctuating part $F_{f x}(r, \theta, z, t)$ which is smaller in magnitude. Here $(r, \theta, z)$ are cylindrica) polar coordinates and $t$ is the time variable. The snall parameter $E$ allows the averaged force $F_{a v}$ to vary in time, but 
slower than the transverse betatron oscillation time-scale; it is also consistent with a finite beam bunch whose length is much greater than its width.

Some considerations for these ( $E z, \varepsilon t$ ) variations in $F_{a v}$ are given eisewhere $\lfloor 7\rfloor$. Even omitting these variations, a "warm-beam" approximation is helpful. Onty those particle orbits are retained whose amplitude of radial excursion (transverse to beam axis) is not greater than about one third of its average radius [6]. Within this model the vlasov $k$ inetic theory for wave disturbances is usually analytically tractable. This is the case provided one has some insight into the relative ordering of critical parameters (such as the equivalent radial mode number measuring how rapidly the mode varies with radius compared to the radial amplitudes of these orbits).

The hydrodynamic model of this paper is introduced in Section II partly in response to the need to identify relevant regimes of radial mode numbers, and partly to initiate the exploration of the nontrivial parameter space in the most efficient manner. If instabilities are found, they are further studied with the kinetic approach. Accordingly, since the electrostatic problem with conducting walls already involve nine parameters, we only make a preliminary examination of resistive wall instabilities while leaving until lat $\cong r$ discussions such topics as electromagnetic effects, etc.

Moreover this hydrodynamic model is a simplified version of the fluid equations derived directly for warm bearns by the analyses of Mark [7j within relevant regimes. Not surprisingly then, the local dispersion relationship of our fluid model (Section II), in appropriate limits, has a close correspondence with the similar relation derived from the Vlisov kinetic equation. The global dispersion relation for electrostatic disturbances (Section III) and numerical results (Section IV-V) of our fluid model are some examples of what 
could be done with this fluid model (sf. also Refs. [8] and [9]). Such discussions are now the "stepping stones" to a more extensive kinetic study of possible relevant instabilities (e.g. cf. Ref. $\lfloor 10\rfloor)$.

One useful result may already be stated for the more general context of beam propagation and transport (including transport in gases). Usually in this subject, more involved problems are resolved using particle code simulations (cf. for example Refs. $\lfloor\backslash\rfloor-\lfloor 4\rfloor$ and $\lfloor l l\rfloor$ for some Heavy-Ion fusion applications). The possibility of using hydrodynamic simulations for certain problems is highly attractive because of the removal of velocity space from the simulation grids. Mark's derivation $\lfloor 7\rfloor$ of the fluid equations can be extended to such problems (incluoing inagnetic fields, relativistic effects, etc.). The recommendation from this paper for simulation of nonrelativistic beams is to use hydrodynamics in the transverse direction but use the drift-kinetic equation in the longitudinal direction (cf. Section III of Ref. $[7]$ ) whenever it is desirable to retain longitudinal Landau damping. For the example of relativistic beams, the particie motions are all at the velocity of light in the first approximation and longitudinal Landau damping is less relevant.

II. THE WRRM BEAM FLUID MUDEL

It is well known that the continuity and momentum moments of Vlasov's equation give exact hydrodynamic equations [cf. Ref. 5 J even though their use poses the question of closure equations for the stresses. The model used here is Dased on retaining three different diagonal stresses $p_{r}, p_{\theta}, p_{z}$, in the $(r, \theta, z)$ directions and no of $f$-diagonal stresses. In particular for closure we assurne a "barotropic fluid" type of adiabatic relation where each 
stress component is a function of dersity. Thus the generalized "enthalpies" per unit mass are

$$
n_{j}=\frac{d p_{j}}{\rho}=a_{j}^{2} \frac{d n}{n}, \quad(j=r, \theta, z)
$$

where $\rho$ and $n$ are the mass and number densities and $a_{j}$ are the "equivalent acoustical speeds" corresponding to each stress component $\mathbf{p}_{j}$.

We may note that in the warm bean kinetic equilibrium of paper $I$, applicable to anharmonic space charge forces, we assumed that particle orbits have radial excursions smaller than dverage orbit radius. Thus in order to retain orbits which have both signs of angular momentum, it is necessary to adopt two separate counter rotating streams. Zero total angular momentum was assumed. This approximation introduces a counter-streaming instability in the azimuthal direction, an unrealistic feature which could be turned of $f$ by decreasing the rotation frequency of each stream.

Each one of the above streams has in addition "temperatures" measured by the velocity dispersions $c_{i}(i=r, \theta, z)$. In our above fluid model, it is natural to identify the acoustic speeds $a_{j}$ with the dispersions $c_{j}$. In the fluid equilibrium, the radial acoustic speed a $a_{\text {ro }}$ is constrained by radial force balance.

Let us introduce the hydrodynamic velocity $\left(v_{r}, v_{\theta}, v_{z}\right)$ which characterizes each of the two counter-rotating streams with opposite sjgns of rotation frequency $s$. The coasting beam equilibrium adopted here has infinite length in the axial or $z$ direction. We need only consider radial force balance involving the equilibrium quantity $\left[\frac{n_{0}}{2}\right]$ ( $n_{0}$ is the total equilitrium beam density of the two streams). Since 


$$
h_{r_{0}}=s\left(a_{r_{0}}\right)^{2} \frac{d n_{0}}{n_{0}}
$$

and

$$
v_{\theta_{0}}=r s(r),
$$

we obtain

$$
s^{2} r-\frac{d h_{r o}}{d r}=v_{0}^{2} r-\frac{1}{r} \int_{0}^{r} \omega_{p}^{2}(r) r d r,
$$

where $s(r)$ is the rotation frequency of the streams each with its awn sign (si). Also $v_{0}{ }^{2} r$ is due to the averaged external focusing force and the $\omega_{p}^{2}$ term represents the space charge of the combinea beam system. The ion plasma frequency $\omega_{p}$ is

$$
\omega_{p}^{2}=\frac{4 \pi n_{0}(r) q^{2}}{m},
$$

where $q$ and $m$ are the ion charge and mass. If the equilibrium beam density $n_{0}$ is constant (simple harmonic space charge farces) then equation ([1-4) is reduced to

$$
s c^{2}-\frac{1}{r} \frac{d h_{r 0}}{d r}=v_{0}^{2}-\frac{1}{2} \omega_{n}^{2} .
$$

Thus when $\omega_{p}^{2}+2 v_{0}^{2},\left[s c^{2}+\frac{1}{r}\left|d n_{r o} / d r\right|\right\rfloor+0$, and the rotation necessarily stops.

This fluid model does not specify the acoustic speeds $a_{\theta}$ or $a_{z}$. From the discussion of neavy-ion accelerators, we do not expect $a_{z}$ and $a_{r}$ to be relatea. But uur kinetic equilibrium results $\left[b_{j}\right.$ inform us that the corresponding kinetic equilibrium implies 


$$
\frac{a_{00^{2}}^{2}}{a_{r 0^{2}}}=\left(\frac{u}{2 s^{2}}\right)^{2}
$$

where

$$
v^{2}=\frac{1}{r^{3}} \frac{d}{d r}\left(1 r^{2} r^{4}\right)
$$

is the generalized becatron frequency. This frequency characterizes the smaller padial (and accompanying $\theta$ ) oscillations of a typical particle orbit of the kinetic equilibriun. This oscillation we called Lo」 betatron vortex oscillation, the latter word being consistent with the terminology of Davidson [11]. Note that in the presence of anharmonic space charge forces, wust distinguish between $\checkmark$ and $\left(z_{i}\right)$. With simple harmonic forces, the betatron frequency $v=2 \Omega$ and $a_{r}=a_{\theta}$.

We now consider wave perturbations with $n_{1}, v_{r l}, v_{\theta 1}, v_{21}$ for each stream defined according to

$$
\begin{aligned}
& n(r, \theta, z, t)=\frac{1}{2} n_{0}(r)+\operatorname{Re}\left[n_{1}(r) e^{i(k \theta+k i z-\omega t)} j,\right. \\
& v_{r}(r, y, z, t)=\operatorname{Re}\left(v_{r 1}(r) e^{i(l(t+k z-\omega t)} J,\right. \\
& v_{\theta}(r, \theta, z, t)=\operatorname{s}(r)+\operatorname{Re}\left[v_{\theta l}(r) e^{i(\nu \theta+k z-\omega t j} j,\right. \\
& v_{z}(r, \theta, z, t)=v_{20}+\operatorname{Re}\left[v_{z]}(r) e^{i(k, t+k z-\omega t)}\right],
\end{aligned}
$$

where $x$ and $k$ are the azimuthal and axial wave number. The continuity equation for these perturbations implies

$$
2(i \bar{\omega}) \frac{n_{1}}{n_{0}}=\frac{I}{r n_{0}} \frac{d}{d r}\left[r n_{0}(r) v_{r i}\right]+i\left(\frac{x}{r^{\prime} \theta 1}+k v_{2\}}\right) \text {, }
$$


where

$$
\bar{\omega}=\omega-k s-k v_{z 0} .
$$

The three momentum equatioris give

$$
\begin{aligned}
& i \bar{\omega} v_{r l}+2 s l v_{r l}=\frac{q}{m} \frac{d \phi_{1}}{d r}+2 \frac{d}{d r}\left(\frac{a_{r}^{2} n_{1}}{n_{0}}\right), \\
& i \bar{\omega} v_{\theta 1}-\frac{v^{2}}{2 s i} v_{r l}=\frac{i l q_{1}}{m r} \Phi_{1}+2 \frac{i l a_{\theta 0}^{2}}{n_{0} n_{1}}, \\
& i \bar{\omega} v_{21}=i k\left(\frac{g}{m} \phi_{1}+2 \frac{a_{20}^{2}}{n_{0}} n_{1}\right) .
\end{aligned}
$$

Eijminating $v_{r l}, v_{\theta l}$, and $v_{z l}$ ir-an equations (II-13) and (II-15)-(II-17) gives an equation in the perturbed density $n_{1}$ and the electric potentiai $\phi_{1}$ :

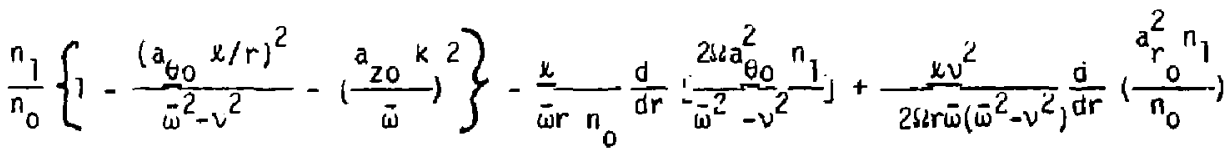

$$
\begin{aligned}
& =-\frac{1}{\omega n_{0} r} \frac{d}{d r} \frac{\bar{\omega} r n_{0}}{\bar{\omega}^{2}-v^{2}}\left[\frac{q}{2 m} \frac{d \phi}{d r}+\frac{d}{d r}\left(\frac{a_{0}{ }^{2} n}{n_{0}}\right)\right] \\
& +\frac{l s_{i}}{r \bar{\omega}\left(\bar{\omega}^{2}-v^{2}\right)}\left[1-\frac{v^{2}}{(2 \omega)^{2}}\right] \frac{q}{m} \frac{d \Phi}{d r} \\
& +\frac{q}{m} \Psi_{l}\left\{\frac{z}{\bar{\omega} r n_{0}} \frac{a}{d r}\left[\frac{\Omega n_{0}}{\omega^{2}-v^{2}}\right]+\frac{(u / r)^{2}}{2\left(\bar{w}^{2}-v^{2}\right)}+\frac{1}{2}\left(\frac{k}{w}\right)^{2}\right\} \text {, }
\end{aligned}
$$


which must be salved in conjuction with Poisson's equation for electrostatic waves

$$
\frac{1}{r} \frac{a}{d r}\left(\frac{d \phi}{d r}\right)-\left(k^{2}+\frac{e^{2}}{r^{2}}\right) \phi_{1}=-4 \pi q \sum_{\operatorname{sgn}(s i)} n_{1} .
$$

The sum over $\operatorname{sgn}(\Omega)$ occurs because Poisson's equation involves the total pertured charge density of the two counter rotating streans.

\section{III.A GLUEAL DISPERSION RELATION}

Equations $(I I-18)-(11-\mid y)$ could in general be solved numerically for beams in a cylindrical conductor (Fig. 1) to determine the eigenvalue $\left(w / v_{0}\right)$. However, the solution depenas on a number of parameters, they are $\left(v / \nu_{c}\right)$, $\left(s / \nu_{0}\right),\left(\omega_{p} / \nu_{0}\right),\left(a_{r} / \nu_{0}{ }_{p}\right\rangle,\left(a_{z} / \nu_{0} R_{p}\right),\left(v_{z 0} / \nu_{Q} R_{p}\right),\left(R_{p} / k_{w}\right)$, and the mode numbers $\left(k_{p}\right), x$ and a radial mode number ootained by solving the eigenvalue problem in the radial direction. Thus it is judicious to first consider the case where the equi?ibrium has a constant plasma density no inside the plasma radius (cf. Fig. 2). This results in harmonic adial forces where $v=23$. The main aovantage of harmonic forces is that equations (II-18) and $(11-) y)$ have solutions in terms of Bessel functions $d_{x}($ Tr) and all that remains of the eigenvalue problem is a pair of simultaneous global dispersion relations; one of these determines the radial mode number $T$ in terms of the frequency $w$ while the other relation determines $w$ itself.

The constancy of the particle density $s_{0}$ imp $\}$ ies $\nu=\angle \Omega$ and $\omega_{p}$ are all constants. If we also assume that $a_{r}$ and $a_{2}$ are constants for $r<R_{p}$, it is easy to see that equations $(11-18\}-(1)-19)$ admit solutions where $n_{3}$ and 
$\Phi_{1}$ are both proportional to $J_{l}(T r)$ where $T$ is a (possibly complex-value radial mode number. Making sucn a substitution gives is

$$
\begin{aligned}
T^{2}\left[1-\sum_{s g n} \frac{\omega_{p}^{2} / 2\left(\bar{\omega}^{2}-v^{2}\right)}{1-\frac{a_{r}{ }^{2} T^{2}}{\bar{\omega}^{2}-v^{2}}-\frac{a_{2}{ }^{2}{ }^{2}}{\bar{\omega}^{2}}}\right] \\
=-k^{2}+\sum_{s g m w} \frac{\omega_{p}{ }^{2} k^{2} / 2 \bar{\omega}^{2}}{1-\frac{a_{r}^{2} T^{2}}{\bar{\omega}^{2}-v^{2}}-\frac{a_{z}^{2} k^{2}}{\bar{\omega}^{2}}}
\end{aligned}
$$

which can be viewed as an equation giving the radial mode number $T$ in $t$ frequency $w$. Especially when $\left(a_{r} T / \nu\right)$ is not large, iterative numerica] solution for $T(\omega)$ is rather straigntforward.

A second equation which determines $w$ is obtained when we matich $t$ solution inside the plasma $\left(r \leq R_{p}\right)$ to the outside solution. Assuming conducting wall at $r=R_{W}>R_{p}$, the boundary condition for solution outside of beam is

$$
\Phi\left(r=R_{w}\right)=-\frac{\omega z}{c k}\left\{\left(\frac{\partial \Phi}{\dot{o} r}\right)_{r=R_{w}}\right.
$$

where $\mathcal{f}$ is the wall impedance in units of $\xi_{0}=377 \mathrm{ohm}$. The wall imped in general, can be a complex number, but for a perfect conducting wall zero. Matching the solutions inside and outside of the beam at the bea surface, we obtain 


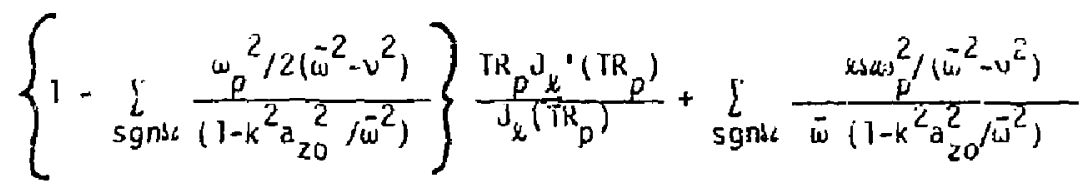

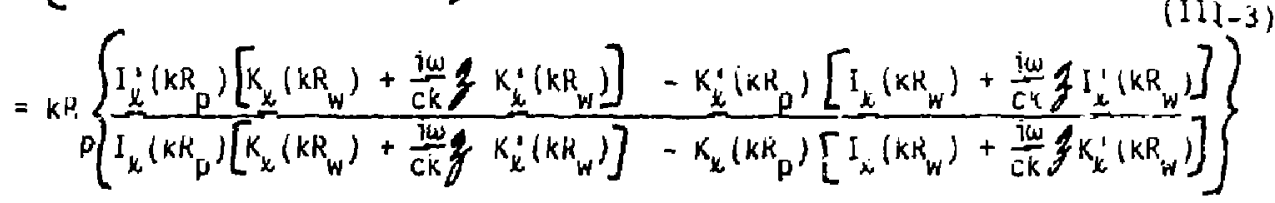

In deriving equ:tion (III-3), we have first assumed icf. Fig. 2; that there is a very thin Doundary layer $\left(R_{D}-\varepsilon\right\rangle<r<\left(R_{D}+\varepsilon\right)$ where the density $n_{0}(r)$ fall: smoothly to zero from its inner constant value for $r<R_{p}-\varepsilon$. In this lajer. we also assumed that $a_{r_{0}}+0$ more rapidly than $n_{0}$ as would be required for a proper warm beam kinetic equilibrium (cf. Ref. [6]).

IV. DISCUSSION OF NUMERICAL RESULTS

A. Beam inside of a Perfect Conducting Pipe For a beam inside of a perfect conducting pipe, equation (1II-1) and (III-3) are solved with $\mathcal{y}=0$.

In Fig. 3 we have plotted the square of the Doppler shifted wave frequency $i_{w-k} v_{\text {a }}$ (relative to beam frame) versus plasina frequency squared, $\omega_{p}^{2}$ of the ions for a typical set of parameters: tne axial wavenumber $k k_{p}=1$, wäl] to piasma radij $R_{W} / R_{p}=2$, az zmuthal wavenumber $x=3$ and several values of the axial oispersion speed parameter $\alpha_{z}=a_{z 0}^{2} / v_{0}^{2} R_{p}^{2}$. These frequencies in the figure are normalizer relative to magnitude of $v_{0}$, tire external force betatran frequency undepressed oy space charge effects. Figure 3 shows that there is a weak instability when $\left(\omega_{p} / \nu_{0}\right)^{2}$ is sufficiently large; the $x=3$ mode being the most unstable. Figure 4 shows the situation witil larger axial wavelengths $\left(k R_{p}=0.2\right)$ which are more comparable to the bunch lengths. 
The insizbility found nere has been shown to be oue to the two counter-rotating streams in this model; when the particle density in one of the streams is made to be zero, the instability vanisnes. That the first unstable mode occurs at $x=3$ in a two-rotating-stream beam was demonstrated previously [12]. The shifting of the threshold density when $\alpha_{z}$ increases as shown in Figs. 3 and 4 has not been discussed before.

The two counter-rotating streams are the artifact of the warm beam model considered here and may not appear in a realistic plasma. However, in reality a spread of rotational angular momentum among beam particles is expected. From that point of view, it is worth while to mention a crude comparison between the present model and the popular kinetic theory model of Kapchinskij and Vladimirskij [13] (abbreviated $K-V$ ). A beam with a $K-V$ distribution reveals two-hump character in both the raoial and azimuthal momenta, which is known to be more unstable thar a plasma with distribution function aecreasing monotically in momenta. In fact, instabilities in the higher radial modes in a beam having a $k-V$ distribution have been found $[14]$. The warm bean model discussed here has two counter-rotatinu streams but no similar two-hump or two-stream behaviors in the radial oirection. Hence we expect that some instabilities found in a beam with $K-V$ distribution will not occur in a warm beam. Both of these models may be different from reality, but they do provide conservative bean current limitations perhaps for somewhat different reasons.

B. Beam in a Pipe with Resistive Surface For a pipe with resistive surface, the wall impedance is taken to be a surely real number. We have observed that for a given nonzero value of 11. this is the case in which the largest growth rate occurs. For 
accelerators in Heavy Ion Inertial fusion, the values of the factor (w/ck) could range from 0 to 0.5 .

When the resistive wall impedance is included, the $x=0$ and $\ell=1$ modes of perturbations, which are stable for $f=0$, become unstable. The first two roots of equations $(111-1)$ and $(111-3)$ for these two modes are found to have the largest imaginary parts. For $y=0.2$, Fig. 5 shows the imaginary parts of the two branches of $x=0, x=1$ and modes. Within the parameter range shown in Fig. $b$, all branches have comparable rauial wave number $T$ and the upper branch of the $x=1$ mode, the kink mode, has the highest growth rate, about a factor of 5 higher than the largest growth rate in $x=0$ mode, the axi-symmetric mode. Note that when the axial acoustic spees $a_{z 0}$ increases, the growth rate in the upper branch of the kink moae and axi-symetric node decrease slightly but the growth rate in the law oranch ot the kink mode increases almost linearly with the pressure. It filight be interesting to recall that the growth rate of the fire hose instability in a nonneutral plasma column has also a linear dependence on the longitudinal pressure.

Not shown in the Fig. 5 are the first two damped branches for $x=0$ and $x=1$ modes among the solutions of equation (III-I) and (III-3). These two branches have negative imaginary parts and are less affected by the variation of $a_{20^{\circ}}^{2}$ Also, a linit that shoulo be mentioned nere is that when $c_{z}$ uinirishes, the upper branch approaches the result in reference $[12\rfloor$ with a resistive boundary.

In concluding the discussion, we notice that within the context of the warm beam model, a hyarodynamic approximation is likely to be nore conservative than the Vlasov-Kinetic description in certain regimes because 
the Landau damping is omitted. However, discussions of a hydrodynamic model gives insight as to the regimes where possible instabilities might lie amidst the nontrivial extent of parareter space in this three dimensional problem. Besides, the discussions of hydrodynamic approximation also help in exploring the limitations of hydrodynariic simulation for beams propagating in gases (cf Section I, last paragraph). The more involved kinetic discussion of the instabilities found in this paper is being pursued, especially on the competitive effects between Landau damping due to the longitudinal energy spread and the resistive kink destabilizing tendancy caused by the axial pressure. The complexity of the kinetic results is due to these cancelling effects, making it worthwhile to first exhibit the destabilizing tendancy by the fluid model without Landau damping. 


\section{REFERENCES}

[1] ERDA Surmer Study of Heavy lons for Inertial fusion, zds. R. 0. B.angenter, W. B. Herrmansfeldt, and L. Simith, Lawrence Berkeley Laboratory Report No. LBL-5534 (1976).

12] Proceedings of the 1933 Heavy Ion Fusion Workshop, ed. L. W. Smith, Brookhaven Nationai Laboratory Report No. BNL-50769 (1977).

[3] Proceedings of the 1978 Heavy Ion Fusion Workshop, ed. R. C. Arnold, Argonne National Laboratory Report No. ANL-79-41 (1978).

[4] Proceedings of the 197y fieavy Ion Fusion Workshop, ed. W. B. Herrmansfeldt, Lawrence Berkeley Laboratory Report No. LBL-10301, Stanford Linear Accelerator Center Report No. SLAC-PUB-2575 (1979).

[5] Mantgamery, D.C. and Tidnan, D.A., "Plasma Kinet ìc Theory," McGraw Hill Book Co., New York (1964).

[6] Mark, J. W-K. and Smith, L., "Heavy Ion Accelerator Plasmas: The Warm Beam Equilibria," Lawrence Livermore National Laboratory Report No. UC10-18947a, Part I, Society for Industrial and Applied Mathematics Journal on Applied Matherratics, in press, (1981). 


\section{ERENCES (continued)}

[7] Mark, J. W-K., "Heavy Ion Accelerator Plasmas: Aaiabatic Theories for Bunched Beams," Lawrence Livermore National Laboratory Report No. UCID-18947 ${ }_{A}$ Society for Industrial and Applied Mathematics Journal on Applied Mathematics, in press, (1981).

[8] Lin, C. C. and Shu, F. H., Astrophys. J., vol. 140, P. 646 (1964).

[9] Lau, Y. Y., Lin, C. C., and Mark, J. W-K., Proc. Natl. Acad. Sci. USA vol. 73, pp. 1379-1381 (1976).

[10] Krafft, G., Mark, J. W-K. and Wang, T. F., "Heavy Ion Accelerator Plasmas: Kinetic Theory of Three-Dimensional Modes with Low Radial Wave Numbers," Lawrence Livermore National Laboratory Report in preparation, (1981).

[11」 Davidson, R. C., "Theory of Nonneutral Plasmas," W. A. Benjamin Press (1974).

[12] Bogema, B. L. and Daviason, R. C., The Phys. of Fluid, 14, 1456 (1971).

[13] Kapchinskij, I. M. and Vladimirskij, V. V., Proc. Int. Conf. on High Energy Accel., CERN, 274 (1959).

[14] Giuckstern, R. L. Proc. Proton Linear Accelerator Laboratory, p. 811 , Batavia, Illinois, (1970). 


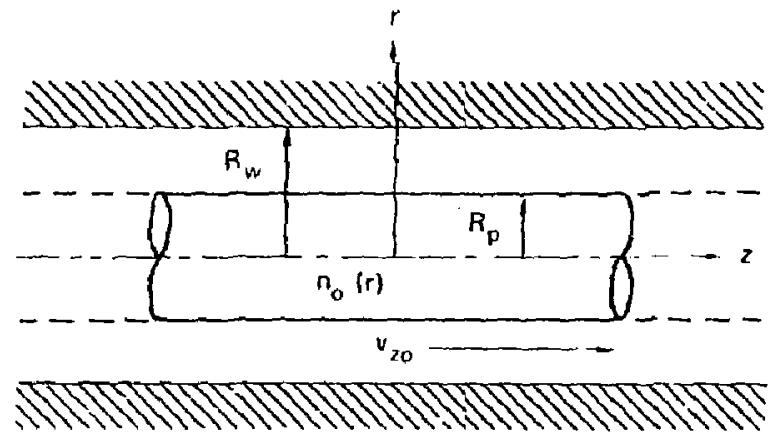

Figure 1. The beam considered here has rafius $R_{p}$ situated within a cylindrical cavity of radius $R_{w}$; its average axial speed is $\nu_{z 0}$ and particle aensity is $n_{0^{\circ}}$ Although not essential, the dean :ength is taken to be infinjte.

$-37-$ 


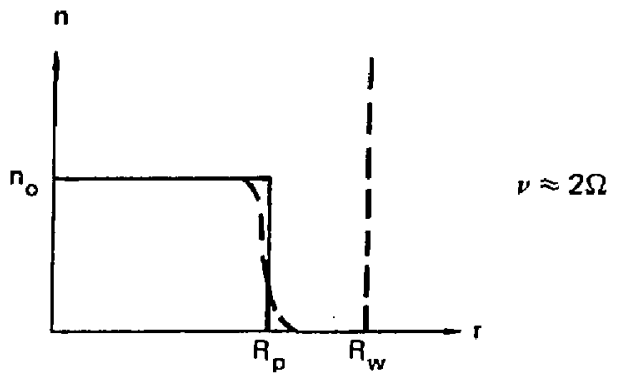

Fjgure 2. The particle density profile adopted for nunerical results in this paper; the dasheo curve is far convenience of matcning bounoary conditions across the plasma radius $R_{p}$. 


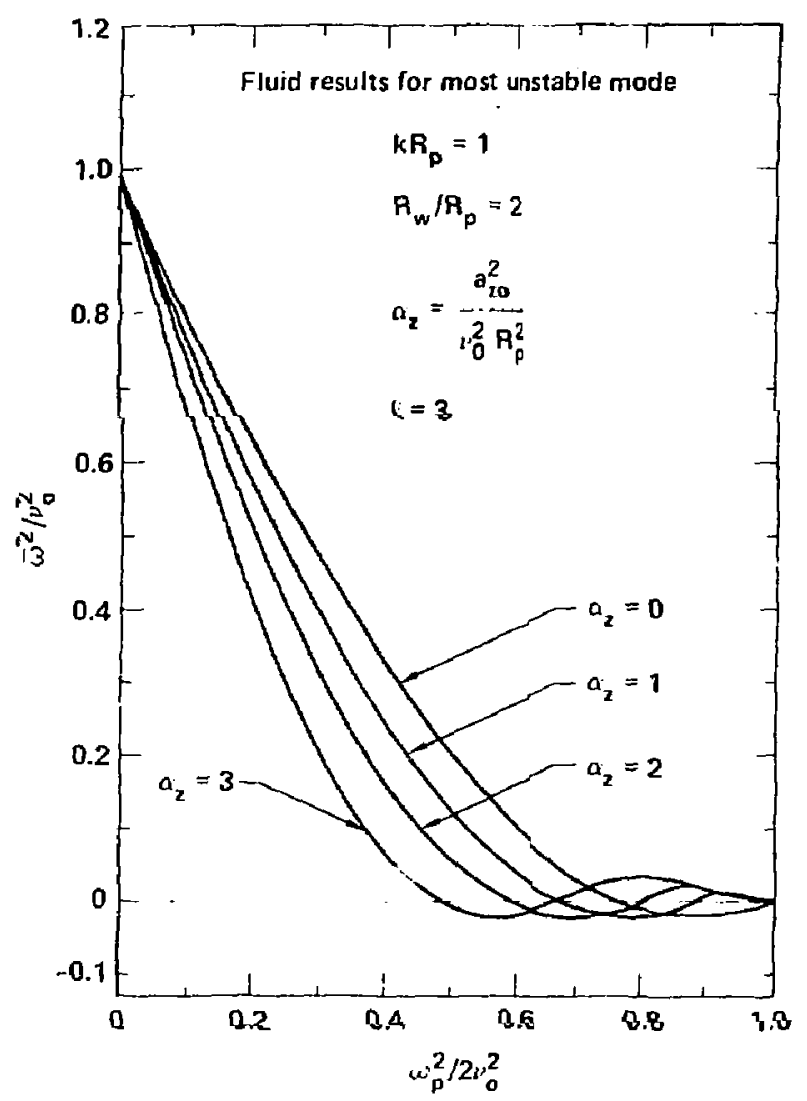

Figure 3. Doppler shifted frequency squared (iw $=w-k_{2} v_{20}$ ) versus beam particle density. Quantity normalizeo by zero density Detatron tune $v_{0}$. 


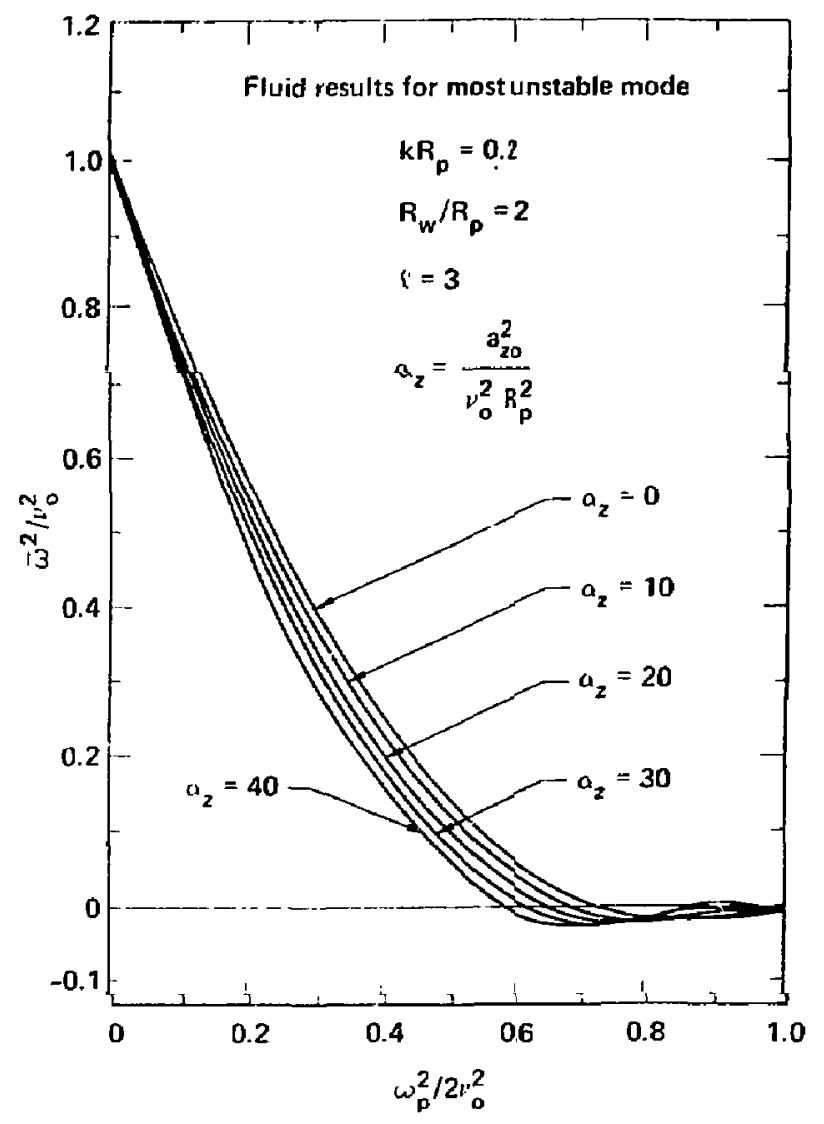

Figure 4. Doppler shifted frequency squareo versus beam particle aensity. 


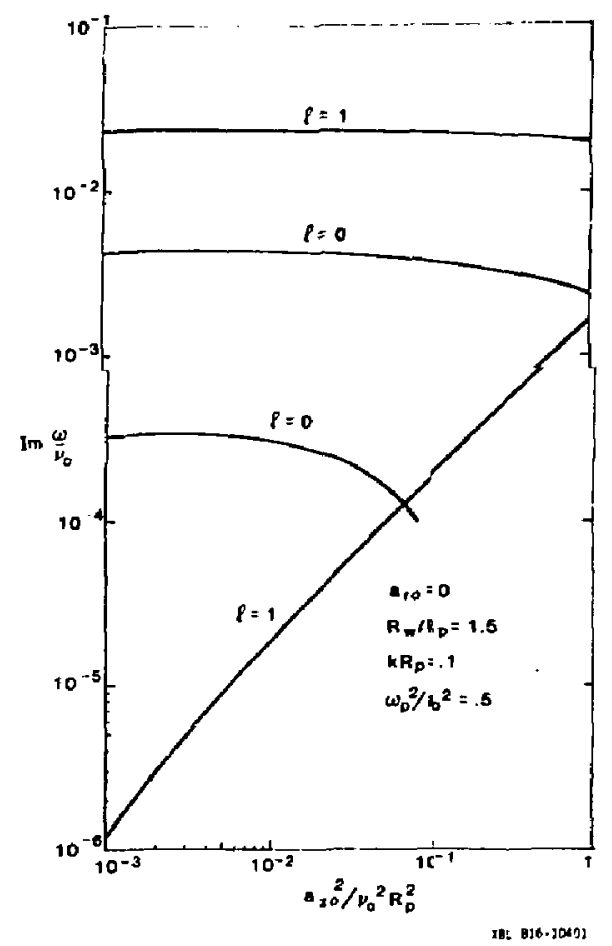

Figure 5. Resistive wall instability growth rates obtained from fluid model, where $y=.02$. 